\title{
Employment of Mapping Technology in Antimicrobial Resistance Reporting in Saudi Arabia
}

\author{
Abdullah A. Alhifany, ${ }^{1}$ Abdullah F. Alqurashi, ${ }^{2}$ Mohamed H. Al-Agamy, ${ }^{3}$ Nasser Alkhushaym, ${ }^{4}$ \\ Faten Alhomoud, ${ }^{5}$ Farah K. Alhomoud, ${ }^{5}$ Thamer A. Almangour ${ }^{6}$ \\ ${ }^{I}$ Clinical Pharmacy Department, College of Pharmacy, Umm Al-Qura University, Makkah; ${ }^{2}$ Department of \\ Geography, Umm Al-Qura University, Makkah; ${ }^{3}$ Microbiology and Immunology Department, College of \\ Pharmacy, King Saud University, Riyadh; ${ }^{4}$ Department of Clinical Pharmacy; Royal Commission Health \\ Services Program; Jubail; ${ }^{5}$ Pharmacy Practice Department, College of Clinical Pharmacy, Imam \\ Abdulrahman Bin Faisal University, Dammam; ${ }^{6}$ Department of Clinical Pharmacy, College of Pharmacy, \\ King Saud University, Riyadh, Saudi Arabia
}

\begin{abstract}
Although Antimicrobial Resistance (AMR) is a worldwide threat, local AMR databases do not exist. Unlike other health disasters, developing containment strategies for AMR cannot be started without a representative, local, updated AMR data. However, Geographical Information Systems (GIS) mapping technology is capable of visualizing AMR data integrated with geographical regions. Due to the absence of AMR databases in Saudi Arabia, we searched Medline and Embase databases from inception until May 28, 2018, including literature that reported AMR data on the most prevalent gram-negative bacterial strains in
\end{abstract}

Correspondence: Abdullah A. Alhifany, Clinical Pharmacy Department, College of Pharmacy, Umm Al-Qura University, 13578 Taif Rd, Makkah, 21955, Saudi Arabia;

Tel.: +966555521820.

E-mail: aahifany@uqu.edu.sa;

Key words: Antimicrobial resistance; Geographical information system; Enterobacteriaceae; Pseudomonas aeruginosa; Acinetobacter baumannii; Saudi Arabia.

Acknowledgments: The authors want to thank the Deanship of Scientific Research at Umm Al-Qura University for supporting this work by Grant Code: 19-MED-1-02-0003. The authors want to thank, as well, King Saud University, Riyadh, Saudi Arabia, for supporting this research project (RSP2020/74). The authors would like to thank also Ibrahima Sall, Reema Alammari, and Ghufran Alhajjaji for their initial help and writing assistance.

Conflict of Interest: The Authors declare no potential conflict of interests.

Received for publication: 23 February 2020.

Accepted for publication: 6 March 2020.

(C) Copyright: the Author(s), 2020

Licensee PAGEPress, Italy

Geospatial Health 2020; 15:868

doi:10.4081/gh.2020.868

This article is distributed under the terms of the Creative Commons Attribution Noncommercial License (CC BY-NC 4.0) which permits any noncommercial use, distribution, and reproduction in any medium, provided the original author(s) and source are credited.
Saudi Arabia. These data were extracted into Microsoft Excel file and inserted into STATA software, version 13 and ArcMap 10.6 software platform for mapping. We found particularly high levels of AMR in Makkah (Mecca), possibly due to high antibiotic consumption because of the influx of pilgrims, with Pseudomonas aeruginosa isolates showing the highest resistance rate against amikacin, aztreonam, cefepime, ceftazidime, ciprolfloxacin, gentamicin, imipenem, meropenem and pipracillin/tazobactam, and Enterobacteriaceae isolates against cefuroxime, ciprofloxacin, ampicillin, imipenem and ertapenem. The cause is, however, multifactorial since Acinetobacter baumannii isolates showed a variable resistance rate throughout the country. The employment of mapping technology in displaying AMR data extracted from published literature is a practically useful approach, and advanced GIS analyses should help stakeholders create containment strategies and allocate resources to slow down the emergence of AMR.

\section{Introduction}

One of the greatest breakthroughs in the last century was the invention of penicillin by Alexander Fleming, which saved thousands of lives threatened by bacterial infections during the Second World War (Lobanovska and Pilla, 2017). Even though the Second World War ended in 1945, another war started, this time between antibiotics and the bacterial tendency to develop resistance. It has been reported that bacterial resistance to antibiotics has resulted in more than 2 million infections and 23,000 deaths annually in the United States alone, costing 55 billion USD in direct and indirect costs (Slayton et al., 2015). Consequently, the US presidential executive order for Combating Antibiotic-Resistant Bacteria was signed in 2014 (Jooma, 2015). The following year saw the launch of the US National Action Plan, which recommends reporting antibiotic use and bacterial resistance to strengthen and monitor the progress of antibiotic stewardship programs in inpatient settings (Slayton et al., 2015).

Antimicrobial Resistance (AMR) is a worldwide health problem causing a major threat to global health (Jooma, 2015; Slayton et al., 2015). In October 2015, the World Health Organization (WHO) launched the Global Antimicrobial Resistance Surveillance System (GLASS) as a global, collaborative initiative to contain the rapid spread of AMR (WHO, 2016).

In February 2017, the WHO declared a critical need for antibi- 
otics to be developed for a list of 12 resistant bacteria and stated that with this accelerated evolution of bacterial resistance to antibiotics, normal medical procedures would be at risk and potentially jeopardize the life of patients (Shrivastava et al., 2018) Despite the absence of a national database pertaining to antibiotic consumption and AMR in Saudi Arabia, there are many published literature references describing the emergence of AMR, yet, they are inconsistently reported (Al-Obeid et al., 2015; Al-Qadheeb et al., 2010; Al Johani et al., 2010; Baadani et al., 2013; Balkhy et al., 2012; Kader and Kumar,
2004; Khan and Faiz, 2016; Memish et al., 2012; Rotimi et al., 1998; Tawfik et al., 2011; Zowawi et al., 2013). In May 2017, Saudi Arabia enrolled in GLASS and submitted 8,219 isolates from 39 surveillance sites, via the Saudi Center for Disease Control (SCDC) (WHO, 2018). Nevertheless, there are many other difficulties surrounding the estimation of AMR in Saudi Arabia, mainly due to the paucity of data with regard to geographical distribution, prevalence and incidence of AMR, making its burden difficult to measure and hindering the development of strategies for its control (Hay et al., 2018).

Table 1. Cumulative collection of samples investigated.

\begin{tabular}{|c|c|c|c|}
\hline Isolate collection (year) & Enterobacteriaceae (no.) & A. baumannii (no.) & P. aeruginosa (no.) \\
\hline 1998 & 414 & & 288 \\
\hline 1999 & 474 & & 301 \\
\hline 2000 & 350 & & 1859 \\
\hline 2001 & 8,239 & & 1283 \\
\hline 2002 & 374 & & 1288 \\
\hline 2003 & 172 & & 1004 \\
\hline 2004 & & & 1478 \\
\hline 2005 & & 49 & 571 \\
\hline 2006 & & 1,490 & \\
\hline 2007 & & 1244 & \\
\hline 2008 & & 106 & \\
\hline 2009 & & 370 & 7,406 \\
\hline 2010 & 120 & 240 & 156 \\
\hline 2011 & 534 & $\sqrt{1}$ & \\
\hline 2012 & & 1383 & \\
\hline 2013 & 202 & 48 & 121 \\
\hline 2014 & 33 & 254 & \\
\hline 2015 & 284 & 17 & 31 \\
\hline Total & 11,196 & 5,312 & 15,791 \\
\hline
\end{tabular}

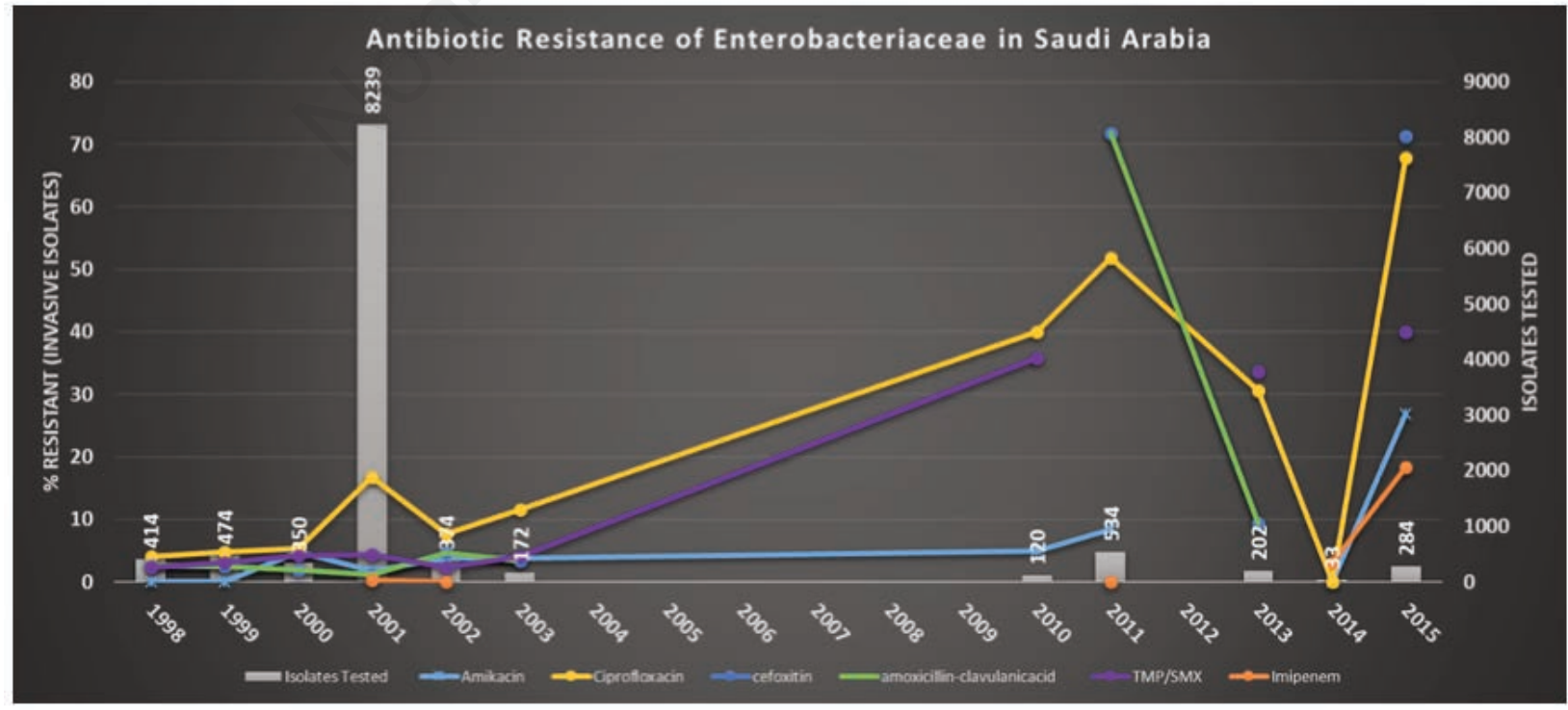

Figure 1. Enterobacteriaceae resistance pattern 1998-to 2015 in Saudi Arabia according to literature search. The grey bars indicate time and number of sample collections. 
Geographical Information Systems (GIS) and mapping techniques, powerful tools that can help visualize local, regional and national disease patterns, have been utilized in primary care research in general and in epidemiological studies (Noble et al., 2012; Samarasundera et al., 2012). Galvin et al. (2013) used GIS to investigate antimicrobial resistance patterns in pathogenic bacteria which highlighted GIS capabilities to visualize epidemiological trends in bacterial pathogens. To the best of our knowledge, GIS has not been used in Saudi Arabia for the purpose of reporting AMR for either public health information or for the management of outbreaks. Thus, this research aims to use the advantage of GIS to map and visualize the characteristic of AMR based on data published in previous research.

\section{Materials and Methods}

Due to the absence of AMR databases in Saudi Arabia, we utilized AMR data published in the literature as reference. We conducted a literature search of the Medline and Embase databases from inception until May 28 ${ }^{\text {th }}, 2018$. The search terms were AMR and the four most prevalent gram-negative bacterial strains in Saudi Arabia: Acinetobacter baumannii, Pseudomonas aeruginosa, Escherichia coli and Klebsiella pneumoniae with the two latter here referred to as Enterobacteriaceae. The AMR-related data were extracted into Microsoft Excel files, which included type of
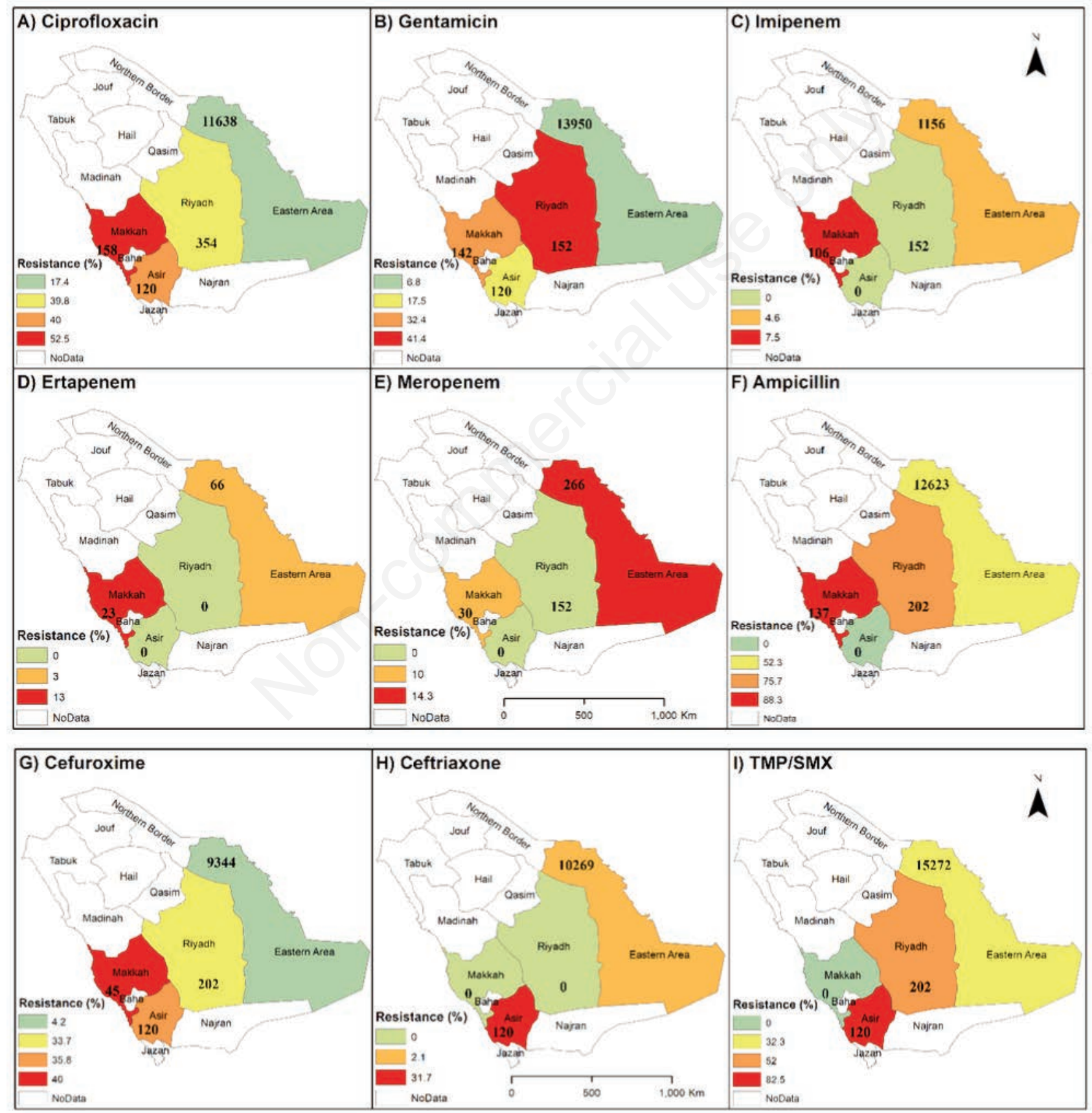

Figure 2. Spatial distribution of the Enterobacteriaceae resistance data in Saudi Arabia. 
bacterial strain, number of isolates tested, sensitivity results, location of the study and time of data collection. The data were pooled and summarized using STATA software, version 13 (https://www.stata.com/), and the spatial view (mapping) was generated using ArcGIS software platform (ESRI, Redlands, CA, USA). The Excel files were inserted into the GIS platform using the map of the Saudi Arabian administrative regions as base layer (feature class). A number of ArcMap tools and functions such as 'merge', 'dissolve' and 'table join' were used to organize and map the collected datasets. Finally, the maps were presented as a percentage of resistance of each bacterial type in the Saudi Arabian regions.

\section{Results}

\section{Enterobacteriaceae}

The Antimicrobial Sensitivity Testing (AST) for a total of 11,196 Enterobacteriaceae isolates were extracted from the published literature between 1998 and 2015. AST data showed an uptrending pattern with respect to resistance against ciprofloxacin, Trimethoprim/Sulfamethoxazole (TMP/SMX), and amikacin. (Table 1 and Figure 1). Demonstration of the spatial distribution of the AST data showed that most were reported from four of Saudi Arabia's 13 administrative regions: the Eastern Region, Riyadh, Asir and Makkah. AMR in the Makkah region was the highest against ciprofloxacin, imipenem, ertapenem, ampicillin and cefuroxime. In the Riyadh Region it was the highest against gentamicin, while the Eastern Region had the highest AMR against meropenem and in Asir Region the bacterial isolates were particularly resistant against ceftriaxone and TMP/SMX (Figure 2).

\section{Pseudomonas aeruginosa}

The AST data for 15,791 $P$. aeruginosa isolates were extracted from the published literature between 1998 and 2015. As can be seen in Figure 3, an up-trending pattern in resistance against all antimicrobials was shown by the AST data. Demonstration of AST data using mapping techniques showed that the majority of isolates were reported from Riyadh, Makkah, Eastern, Asir and Hail regions. Figure 4 shows that AMR in the Makkah Region was the highest against all reported antimicrobials.

\section{Acinetobacter baumannii}

The AST data for 5,312 Acinetobacter baumannii isolates were extracted from published literatures between 1998 and 2015. The AST data regarding these isolates showed an up-trending pattern in resistance against all antimicrobials (Figure 5). Demonstration of AST data using mapping techniques showed that most isolates were reported from the Riyadh and Eastern regions. In this case, the AMR data were the highest against amikacin, ciprofloxacin, and imipenem in Madinah region, and against gentamicin and meropenem in Riyadh region (Figure 6).

\section{Discussion}

To our knowledge, this is the first study to use mapping techniques to represent AMR data at regional levels in Saudi Arabia. In spite of the inconsistency of the data published, regarding the number of bacterial isolates tested, their regional origin, and the time of their collection, it is very obvious that AMR is a growing trend since the last few years. There was variability in the AMR data among regions, which could indicate differences in resistant strains, practice pattern, and prescribing aptitude of local doctors.

Establishing a national database that reports the prevalence of AMR and antibiotics consumption on a real-time basis at regional levels in Saudi Arabia would be an essential first step to allocate resources, detects outbreaks, and supports antimicrobial stewardship programs. The occurrence of AMR in bacterial pathogens has been illustrated and analysed through maps by agencies like European Centre for Disease Prevention and Control (ECDC) where antimicrobial consumption in thirty European countries are

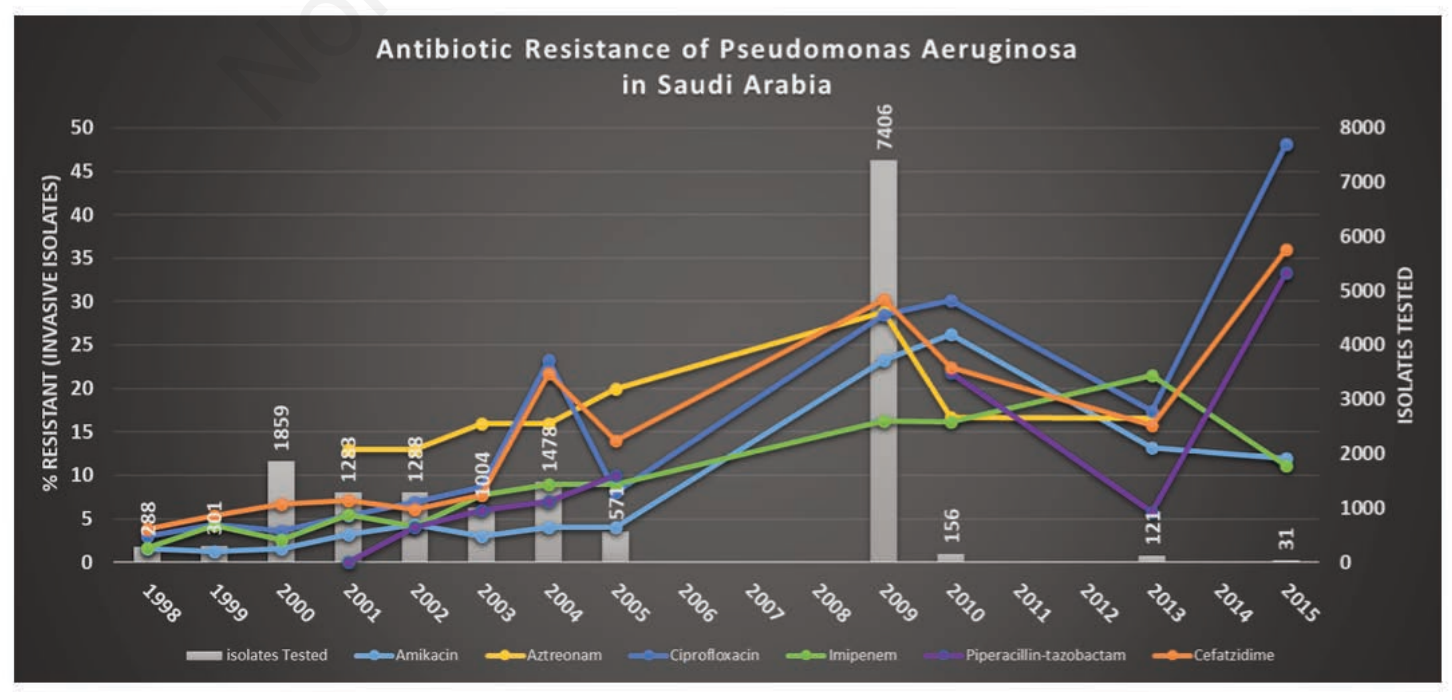

Figure 3. Pseudomonas aeruginosa resistance pattern 1998 to 2015 in Saudi Arabia according to literature search. The grey bars indicate time and number of sample collections. 
reported on a regular basis (Weist and Högberg, 2016). In another research study, using visual and statistical evidence, Callaghan et al. (2009), established the effectiveness of GIS acknowledging that "its unique ability to integrate a large range of datasets in a common framework facilitating in the spatial and non-spatial analysis of disease events adds a different dimension to disease analysis and surveillance". GIS has also been utilized in other research studies in other regions to investigate methicillin-resistant Staphylococcus aureus (Tirabassi et al., 2005), Streptococcus pneumonia (Mouro et al., 2011) and E. coli.(Kiffer et al., 2011).

This study, the first to investigate antimicrobial resistance trends in Saudi Arabia using GIS, aimed to explore and use GIS technology applications to gain insight of the extent of AMR in Saudi Arabian regions, and to come up with surveillance and monitoring strategies using GIS derived products (maps, spatial and temporal analyses, etc.) that could help address current and future outbreaks more quickly and efficiently. Although GIS, through the mapping techniques used in this study, presents the best method to visualize the characteristic of AMR in Saudi Arabia, our research did not utilize an advanced GIS analysis due to limitations in the availability of data. Advanced analysis includes, for example, spatial interpolation such as Inverse Distance Weighted (IDW), Kriging, natural neighbour, spline and trend techniques, which may be considered in the future.

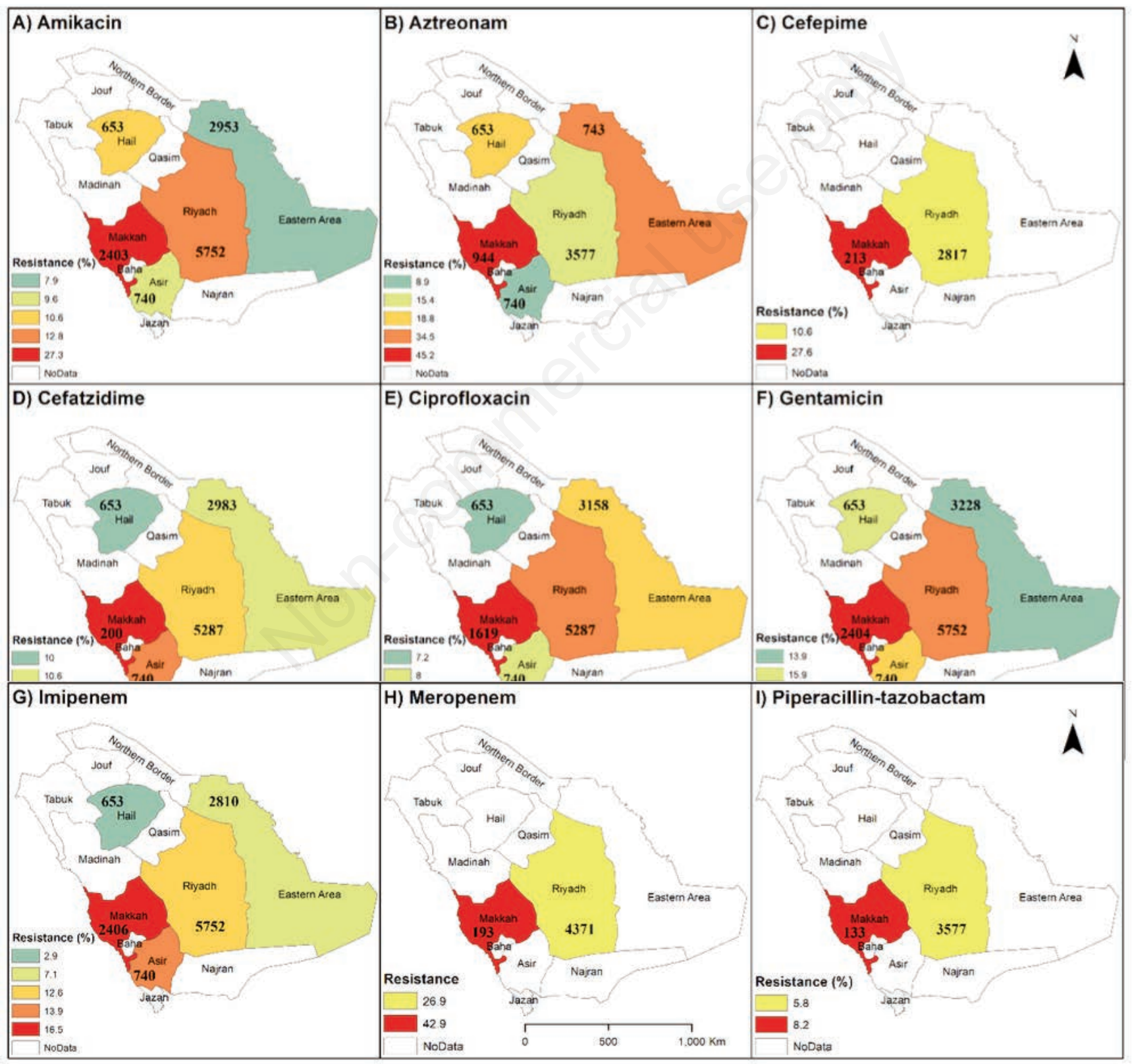

Figure 4. Spatial distribution of the Pseudomonas aeruginosa resistance data in Saudi Arabia. 


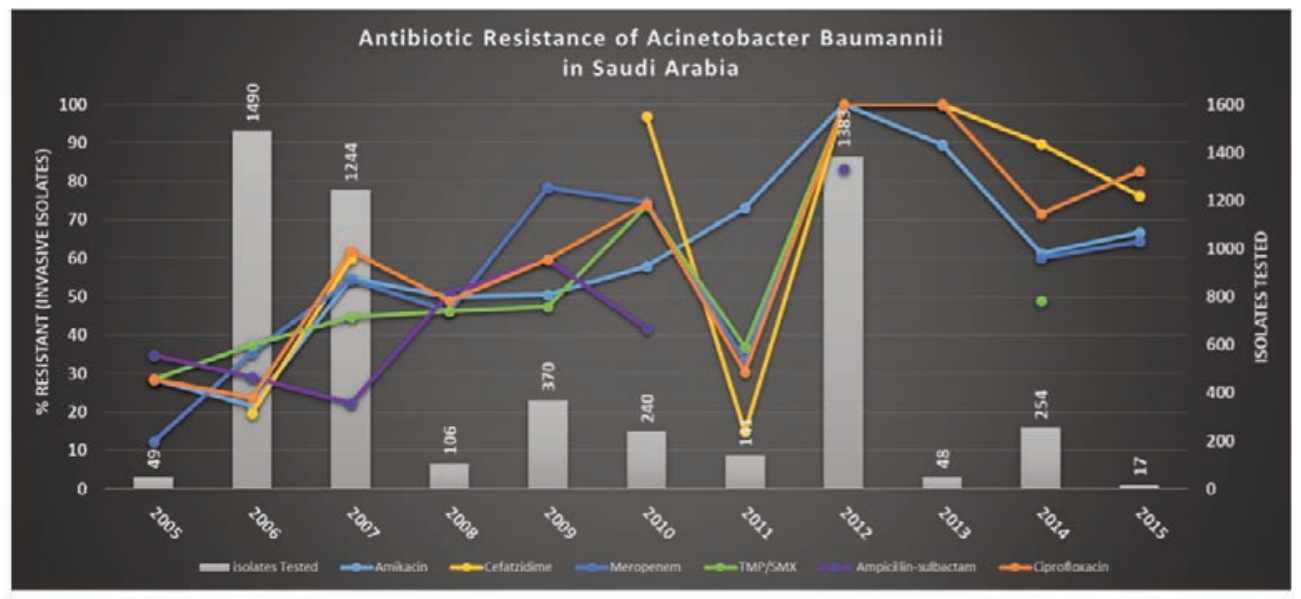

Figure 5. Acinetobacter baumannii resistance pattern 1998 to 2015 in Saudi Arabia according to the literature search. The grey bars indicate time and number of sample collections.

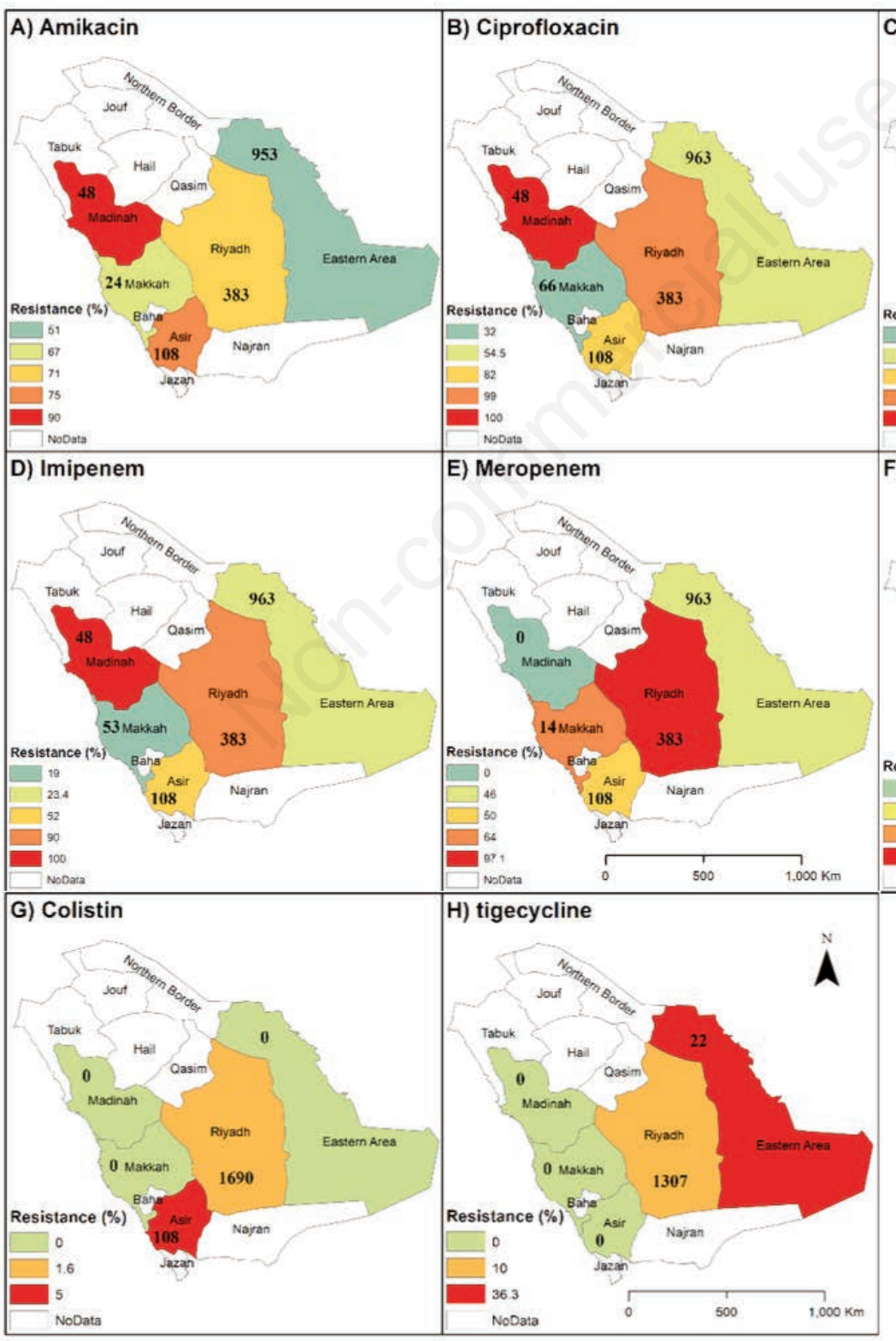

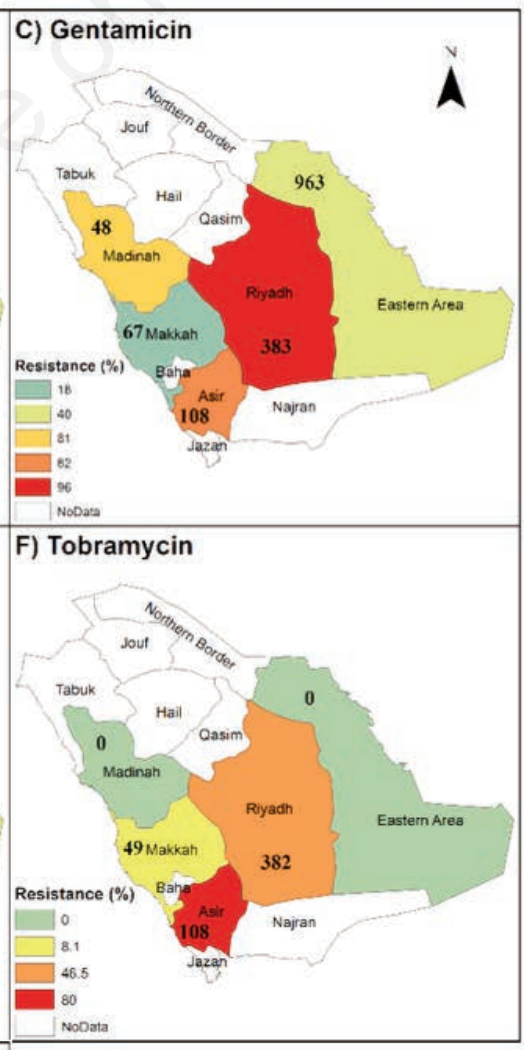

NoData
Figure 6. Spatial distribution of Acinetobacter baumannii resistance data in Saudi Arabia. 
Using such techniques would be worthwhile to improve our understanding regarding spatial trends and pattern recognition of AMR levels in Saudi Arabia. Incorporating different environmental and anthropogenic variables such as wastewater, landfill locations, population number and density, distance from roads and distance from healthcare locations into GIS platform may also be valuable to understand the cause and effect of AMR in Saudi Arabia. Once such data can be obtained, these ideas will be targeted in future research. Nevertheless, the results presented in this research have indicated an effective approach by clearly showing the distribution of AMR in the Saudi Arabian regions between 1998 and 2015.

\section{Conclusion}

The employment of mapping technology in displaying AMR data extracted from published literature shows a high resistance rate in Makkah, which could be attributed to the high antibiotic consumption due to multiple factors, including the high influx of pilgrims. Advanced GIS analyses are expected to help stakeholders create containment strategies and allocate resources to slow down the emergence of AMR.

\section{References}

Al-Obeid S, Jabri L, Al-Agamy M, Al-Omari A, Shibl A, 2015. Epidemiology of extensive drug resistant Acinetobacter baumannii (XDRAB) at Security Forces Hospital (SFH) in Kingdom of Saudi Arabia (KSA). J Chemother 27:156-62. doi:10.1179/1973 947815y.0000000019

Al-Qadheeb NS, Althawadi S, Alkhalaf A, Hosaini S, Alrajhi AA, 2010. Evolution of tigecycline resistance in Klebsiella pneumoniae in a single patient. Ann Saudi Med 30:404-7. doi:10.4103/02564947.67087

Al Johani SM, Akhter J, Balkhy H, El-Saed A, Younan M, Memish Z, 2010. Prevalence of antimicrobial resistance among gram-negative isolates in an adult intensive care unit at a tertiary care center in Saudi Arabia. Ann Saudi Med 30:364-9. doi:10.4103/02564947.67073

Baadani AM, Thawadi SI, El-Khizzi NA, Omrani AS, 2013. Prevalence of colistin and tigecycline resistance in Acinetobacter baumannii clinical isolates from 2 hospitals in Riyadh Region over a 2-year period. Saudi Med J 34:248-53.

Balkhy HH, El-Saed A, Al Johani SM, Francis C, Al-Qahtani AA, AlAhdal MN, et al., 2012. The epidemiology of the first described carbapenem-resistant Klebsiella pneumoniae outbreak in a tertiary care hospital in Saudi Arabia: how far do we go? Eur J Clin Microbiol Infect Dis 31:1901-9. doi:10.1007/s10096-011-1519-0.

Hay SI, Rao PC, Dolecek C, Day NPJ, Stergachis A, Lopez AD, Murray CJL, 2018. Measuring and mapping the global burden of antimicrobial resistance. BMC Med 16:78. doi:10.1186/s12916018-1073-z.

Jooma S, 2015. Executive action to combat the rise of drug-resistant bacteria: is agricultural antibiotic use sufficiently addressed? Journal of Law and the Biosciences 2:129-38. doi:10.1093/ $\mathrm{jlb} / \mathrm{lsv} 005$.

Kader AA, and Kumar AK, 2004. Prevalence of extended spectrum beta-lactamase among multidrug resistant gram-negative isolates from a general hospital in Saudi Arabia. Saudi Med J 25:570-4.

Khan MA, Faiz A, 2016. Antimicrobial resistance patterns of Pseudomonas aeruginosa in tertiary care hospitals of Makkah and
Jeddah. Ann Saudi Med 36:23-8. doi:10.5144/0256-4947.2016.23

Kiffer CR, Camargo EC, Shimakura SE, Ribeiro PJ, Jr., Bailey TC, Pignatari AC, Monteiro AM, 2011. A spatial approach for the epidemiology of antibiotic use and resistance in community-based studies: the emergence of urban clusters of Escherichia coli quinolone resistance in Sao Paulo, Brasil. Int J Health Geogr 10:17. doi:10.1186/1476-072X-10-17

Lobanovska M, Pilla G, 2017. Penicillin's Discovery and Antibiotic Resistance: Lessons for the Future? Yale J Biol Med 90:135-45.

Memish ZA, Shibl AM, Kambal AM, Ohaly YA, Ishaq A, Livermore DM, 2012. Antimicrobial resistance among non-fermenting Gramnegative bacteria in Saudi Arabia. J Antimicrob Chemother 67:1701-5. doi:10.1093/jac/dks091.

Mouro AK, Koga PC; Monteiro AM; Camargo EC, Pignatari AC, 2011. Spatial exploration of Streptococcus pneumoniae clonal clustering in Sao Paulo, Brazil. Braz J Infect Dis 15:462-6.

Noble D, Smith D, Mathur R, Robson J, Greenhalgh T, 2012. Feasibility study of geospatial mapping of chronic disease risk to inform public health commissioning. BMJ Open 2:e000711. doi:10.1136/bmjopen-2011-000711

Rotimi VO, al-Sweih NA, Feteih J, 1998. The prevalence and antibiotic susceptibility pattern of gram-negative bacterial isolates in two ICUs in Saudi Arabia and Kuwait. Diagn Microbiol Infect Dis 30:53-9.

Samarasundera E, Walsh T, Cheng T, Koenig A, Jattansingh K, Dawe A, Soljak M, 2012. Methods and tools for geographical mapping and analysis in primary health care. Prim Health Care Res Dev 13:10-21. doi:10.1017/S1463423611000417.

Shrivastava SR, Shrivastava PS, \& Ramasamy J. 2018. World health organization releases global priority list of antibiotic-resistant bacteria to guide research, discovery, and development of new antibiotics. J Med Soc 32:76.

Slayton RB, Toth D, Lee BY, Tanner W, Bartsch SM, Khader K, et al., 2015. Vital Signs: Estimated Effects of a Coordinated Approach for Action to Reduce Antibiotic-Resistant Infections in Health Care Facilities - United States. MMWR. Morbidity and Mortality Weekly Rep 64:826-31. Available from: https://www.ncbi.nlm. nih.gov/pubmed/26247436

Tawfik AF, Alswailem AM, Shibl AM, and Al-Agamy MH. 2011. Prevalence and genetic characteristics of TEM, SHV, and CTX-M in clinical Klebsiella pneumoniae isolates from Saudi Arabia. Microb Drug Resist, 17(3), 383-388. doi:10.1089/ mdr.2011.0011

Tirabassi MV, Wadie G, Moriarty KP, Garb J, Konefal SH, Courtney RA, ... Wait R. 2005. Geographic information system localization of community-acquired MRSA soft tissue abscesses. J Pediatr Surg, 40(6), 962-965; discussion 965-966. doi:10.1016/j.jpedsurg. 2005.03.010

Weist K, and Högberg LD. 2016. ECDC publishes 2015 surveillance data on antimicrobial resistance and antimicrobial consumption in Europe. Eurosurveillance, 21(46).

WHO, 2016. National antimicrobial resistance surveillance systems and participation in the Global Antimicrobial Resistance Surveillance System (GLASS): a guide to planning, implementation, and monitoring and evaluation. Available from: https:/apps.who.int/ iris/handle/10665/251554

WHO, 2018. Global antimicrobial resistance surveillance system (GLASS) report-early implementation 2016-2017. Available from:https://www.who.int/glass/resources/publications/early-implementation-report/en/

Zowawi HM, Balkhy HH, Walsh TR, and Paterson DL. 2013. betaLactamase production in key gram-negative pathogen isolates from the Arabian Peninsula. Clin Microbiol Rev, 26(3), 361-380. doi:10.1128/cmr.00096-12 